\title{
QUELQUES ASPECTS DE L'ALIMENTATION DE DEUX POISSONS PLANCTONOPHAGES DU LAC LÉMAN : LE CORÉGONE (COREGONUS SCHINZII PALEA CUV. ET VAL.) ET LE GARDON (RUTILUS RUTILUS (L.))
}

\author{
D. PONTON (1), D. GERDEAUX (2)
}

(1) Seenforschungslaboratorium der EAWAG/ETH CH-6047 KASTANIENBAUM, Suisse.

(2) Station d'Hydrobiologie Lacustre, INRA BP $11 \mathrm{~F}, 74203$ THONON-LES-BAINS, France.

Reçu le 23 novembre 1987

Accepté le 17 mars 1988

Received 23 November, 1987 Accepted 17 March, 1988

\section{RÉSUMÉ}

Dans le but de comparer leur alimentation, des corégones et des gardons ont été capturés à l'aide de chaluts pélagiques (gardons seulement), de filets maillants pélagiques et de fond dans le lac Léman entre décembre 1983 et juillet 1985. En zone pélagique, les deux espèces sont bien des planctonophages stricts. Ils y partagent les mêmes ressources trophiques entre juin et octobre. Les proies principales ingérées sont alors Daphnia sp. puis surtout Leptodora kindtii et Bythotrephes longimanus. Il est montré qu'il n'est pas possible de conclure à une compétition alimentaire entre ces deux espèces de poissons faute de données suffisantes sur la biologie de leurs proies.

\section{SOME ASPECTS OF THE FOOD OF TWO PLANCTONOPHAGIC FISHES IN LEMAN LAKE : \\ THE WHITEFISH (COREGONUS SCHINZII PALEA CUV. AND VAL.) AND THE ROACH (RUTILUS RUTILUS (L.))}

\section{SUMMARY}

In order to compare their food, whitefish and roach were captured with trawls (roach only) and pelagic and bottom gill nets in Leman Lake from June 1984 to July 1985. In the pelagic area, the two species are strictly planctonophagic. They share the same trophic ressources from June to October. The principal eaten preys are Daphnia sp. then particularly Leptodora kindtii and Bythotrephes longimanus. The conclusion of a competition between these two species of fishes is not possible on account of the lack of data on the biology of their principal preys.

\section{INTRODUCTION}

Dans le lac Léman, parmi les six espèces principales de poissons (corégone, truite, omble chevalier, perche, gardon et lotte), deux sont planctonophages à l'état adulte: le corégone du Léman ou Palée (Coregonus schinzii palea Cuv. et Val) et le gardon (Rutilus rutilus (L.)).

Le corégone est un Salmonidé de bonne valeur commerciale dont l'importance des captures par la pêche professionnelle varie, suivant les années, entre 50 et 100 tonnes pour tout le lac. Le gardon, habituellement littoral et benthophage, présente dans le Léman un comportement microphage et pélagique comme dans d'autres lacs en cours d'eutrophisation (BOHL, 1982 ; ROTH, 1969. Ayant colonisé tout le lac, il est une gêne pour la pêche professionnelle du fait du manque de débouchés commerciaux.

Dans le cadre de l'étude du peuplement ichtyologique du lac Léman, il était nécessaire de préciser deux points importants:

- Le caractère strict de la planctonophagie du corégone du Léman. La très forte croissance de ce poisson dans ce lac (CHAMPIGNEULLE et al., 1983), ainsi que la grande plasticité du genre Coregonus vis-à-vis de son alimentation (JACOBSEN, 1982) pouvaient laisser des doutes à ce sujet.

- La place exacte du gardon dans le réseau trophique lémanique. Les travaux fragmentaires réalisés sur son régime alimentaire dans ce lac (CHEVALIER, 1980) ne permettaient pas d'évaluer dans quelle mesure le spectre alimentaire de cette espèce et celui du corégone pouvaient se chevaucher. 


\section{MATÉRIELS ET MÉTHODES}

\subsection{Méthodologies d'échantillonnage}

Plusieurs types d'engins ont du être employés pour capturer les poissons.

\subsubsection{Les chaluts pélagiques}

Deux types de chaluts pélagiques ont été successivement utilisés:

- Un chalut pélagique de $12 \times 4$ mètres d'ouverture. Tracté par deux bateaux, il est employé en eaux intérieures en R.F.A. (STEINBERG et DAHM, 1974). Les mailles des nappes qui le constituent vont de $40 \mathrm{~mm}$ à $25 \mathrm{~mm}$ nœud à nœud pour la poche terminale.

- Un chalut pélagique de 2,5 × 2,5 mètres d'ouverture. Ce chalut à barres, employé par l'EAWAG de Kastanienbaum (GEIGER et al., 1985), a été utilisé à partir de l'automne 1984. Constitué de nappes dont les mailles vont de $30 \mathrm{~mm}$ à $5 \mathrm{~mm}$ nœud à nœud pour la poche terminale, il est muni d'une sonde permettant de connaitre sa profondeur d'immersion. Tiré par un seul bateau, il nous a permis de capturer régulièrement des poissons entre 0 et 50 mètres de profondeur.

II est rapidement apparu que seuls les gardons pouvaient être régulièrement capturés à l'aide de ces chaluts. Les corégones, du fait de leur faible abondance dans ce lac de grandes dimensions, ont été obtenus à partir des captures de pêcheurs professionnels.

\subsubsection{La pêche professionnelle de corégones}

Cette pêche, surtout active en èté, s'effectue à l'aide de filets dérivants appelés "grands pics", de 120 mètres de long et d'une hauteur maximale de 18 mètres. La maille minimale légale est de $50 \mathrm{~mm}$. Groupés par 6 ou 12, ces filets, disposés en zone pélagique à une profondeur de 3 à 15 mètres sous la surface, restent au lac 8 à 12 heures durant la nuit.

Les problèmes liés à un èchantillonnage effectué grâce à de tels engins sont nombreux. Tout d'abord, le long temps de pêche permet une poursuite des processus de digestion. Ensuite, la structure de taille, et donc d'âge, de l'échantillon obtenu est liée aux contraintes réglementaires (taille minimale légale de capture des corégones : $300 \mathrm{~mm}$ ). Enfin, l'approvisionnement ne peut se faire régulièrement (dates de fermeture administrative de la pẽche aux Salmonidés et report de l'effort de pêche à certaines périodes de l'année sur d'autres espèces).

\subsubsection{Les filets maillants de fond}

Des filets de mailles allant de 40 à $50 \mathrm{~mm}$ ont été utilisés en zone littorale lors de la reproduction des corégones (c'est-à-dire de la fin décembre à début janvier). Des gardons ont été capturés mensuellement dans la même zone (mailles de 20 à $45 \mathrm{~mm}$ ) à partir de janvier 1985. Ces filets restaient une nuit au lac.

\subsection{4. Échantillonnage du zooplancton}

Le suivi de la dynamique du zoop̉lancton dans le Léman est abordé dans le cadre des travaux de l'Institut de Limnologie de Thonon-les-Bains. Des prélèvements bi-mensuels ou hebdomadaires sont effectués régulièrement en deux points du lac de - 50 mètres à la surface avec un filet de 200 microns de vide de maille pour les Entomostracés (BALVAY et al., 1984).

\subsection{Protocoles d'échantillonnage}

- Captures au chalut : après détection des poissons à l'aide de l'échosondeur, des chalutages ont lieu jusqu'à obtenir une quantité suffisante d'individus. Chaque poisson est individualisé, son tube digestif est immédiatement fixé au formol à cinq pour cent. Ces captures ont eu lieu autant que possible avec une fréquence mensuelle.

- Captures aux pics (corégones et gardons) et aux filets de fond: la remontée des filets s'effectue avant le lever du soleil, les tubes digestifs des poissons capturés sont fixés immédiatement au formol à cinq pour cent.

\subsection{Traitement des échantillons}

\subsection{1. Échantillons de zooplancton}

Les valeurs de l'abondance des différents taxons ont été regroupées en classes d'abondance (FRONTIER, 1969). La cotation établie sur la base d'une progression géométrique de raison 4,3 s'est avérée apte à définir suffisamment de classes pour rendre compte des variations observées (Tableau I). La détermination des taxons est arrêtée au niveau générique sauf pour Bythotrephes longimanus et Leptodora kindtii.

(1) EAWAG : Eidgenössiche Anstalt für Wasserversorgung, Abwasserreinigung und Gewässerschutz : Institut fédéral pour l'aménagement, l'épuration et la protection des eaux. 


\begin{tabular}{|c|c|c|c|c|}
\hline \multirow[t]{2}{*}{ COTES } & \multicolumn{2}{|c|}{ EFEECTIFS } & \multirow[t]{2}{*}{ COTES } & EFFECT IFS \\
\hline & par & $\mathrm{m}^{3}$ & & par $\mathrm{m}^{3}$ \\
\hline 0 & & 0 & & \\
\hline 1 & 1 & à 3 & & \\
\hline & & & 1.5 & 3 ou 4 \\
\hline 2 & 4 & 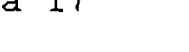 & 2.5 & environ 18 \\
\hline 3 & 18 & à 80 & 35 & onuiron 80 \\
\hline 4 & 80 & à 350 & U. & 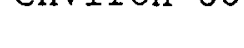 \\
\hline & & & 4.5 & env. \\
\hline 5 & 350 & à 1500 & 55 & 1500 \\
\hline 6 & 1500 & à 6500 & 0.0 & env. \\
\hline & & & 6.5 & env. \\
\hline 7 & 6500 & à 30000 & 75 & \\
\hline 8 & 30000 & à 120000 & 1.0 & C118. 5000 \\
\hline
\end{tabular}

Tableau 1: Définition des classes d'abondance d'après FRONTIER (1969).

Table I : Indices of abundance.

\subsubsection{Analyse des contenus stomacaux}

Les organismes présents dans l'estomac (premier tiers du tube digestif pour les gardons) sont comptés à la loupe binoculaire (grossissement $X 32$ ) si le degré de lyse le permet. La quantité de matériel ingéré nécessite parfois un sous-échantillonnage: la totalité du bol alimentaire est alors homogénéisée dans un grand volume d'eau, le tirage au hasard des sous-échantillons, trois au minimum, est vérifié grâce au test de Fisher (ELLIOTT, 1977).

Une simple énumération des proies ingérées présente l'inconvénient de ne pas tenir compte de leur volume ou de leur poids (LAUZANNE, 1976). Pour y remédier, un facteur de conversion, proportionnel à la gamme de taille du taxon-proie considéré, a été appliqué aux résultats numériques (Tableau II). La composition volumétrique de l'alimentation d'origine zooplanctonique peut ainsi être approchée (PONTON et STROFFEK, 1987).

\begin{tabular}{|c|c|c|}
\hline TAXON & $\begin{array}{l}\text { POIDS DO TAXON } \\
\mu g \text { mat. sòche }\end{array}$ & $\begin{array}{l}\text { FACTEUR DE } \\
\text { CONVERSION }\end{array}$ \\
\hline Bosmina sp. & environ 2 & $x \quad 1$ \\
\hline $\begin{array}{l}\text { Cyclopides } \\
\text { C4 }_{4} \text { àd. }\end{array}$ & $7 \dot{a} 18$ & $\times 5$ \\
\hline $\begin{array}{l}\text { Calanides } \\
\text { C4 à Ad. }\end{array}$ & 5 à 12 & $\times 5$ \\
\hline Daphnia sp. & environ 20 & $\times 10$ \\
\hline Leptodera sp. & 100 a 150 & $\times 50$ \\
\hline Bythotrephes sp. & 50 a 150 & $\times 50$ \\
\hline
\end{tabular}

Tableau II : Facteurs de conversion appliqués aux résultats numériques pour les six taxonsproies zooplanctoniques principaux.

Table II : Conversion factors associated with the six principal zooplanctonic preys. 
A partir de ces données, un pourcentage de recouvrement des spectres d'utilisation de la nourriture zooplanctonique par le corégone et le gardon a été déterminé à l'aide de l'indice de similitude proposé par RENKONEN (1938 in HURLBERT, 1978) défini ainsi :

$C_{i k}=1-0,5\left(\sum k \quad I p_{1 k}-p_{j k}\right)$

avec $p_{1 k}$ et $p_{1 k}$, quantités relatives en volume de ressource de catégorie $k$ utilisée respectivement par les espèces $i$ et $j$.

\section{RÉsULTATS}

L'évolution du nombres d'estomacs et de tubes digestifs vides au cours du temps est présentée pour les corégones (Tableau III), pour les gardons capturés par un chalut en zone pélagique (Tableau IV) et pour les gardons capturés à l'aide de filets maillants en toute zone (Tableau V). Les résultats sont présentés toutes cohortes confondues. Ces résultats font état, pour les deux espèces, d'un nombre élevé de tubes digestifs entièrement vides en période hivernale. En mars et avril, les corégones reprennent leur activité alimentaire plus tôt que les gardons.

\begin{tabular}{|c|c|c|c|c|c|c|c|}
\hline \multirow[t]{2}{*}{ DATE } & \multirow[t]{2}{*}{$\mathbf{N}$} & \multirow{2}{*}{$\begin{array}{l}\text { GAMME } \\
\text { DE } \\
\text { TAILLES } \\
\text { Min-Max }\end{array}$} & \multicolumn{2}{|c|}{$\begin{array}{l}\text { ESTOMACS } \\
\text { VIDES }\end{array}$} & \multicolumn{2}{|c|}{$\begin{array}{l}\text { TUBES } \\
\text { DIGEST IFS } \\
\text { PIDES }\end{array}$} & \multirow{2}{*}{$\begin{array}{l}\text { COMPTAGE } \\
\text { DE.S PROIES } \\
\text { INGEREES } \\
\text { POSSIBLE }\end{array}$} \\
\hline & & & $\mathbf{N}$ & $x$ & $\mathbf{N}$ & $x$ & \\
\hline Dec. 83 & 31 & $3.35-496$ & 3 & 9.7 & 2 & 6.5 & NON \\
\hline $27 / 04 / 84$ & 18 & $383-456$ & 5 & 27.8 & 0 & 0 & NON \\
\hline $7 / 07 / 84$ & 32 & $400-500$ & 0 & 0 & 0 & 0 & OUI \\
\hline $3 / 08 / 84$ & 14 & $367-472$ & 0 & 0 & 0 & 0 & OUI \\
\hline $23 / 08 / 84$ & 39 & $393-485$ & 1 & 2.6 & 0 & 0 & OUI \\
\hline $21 / 09 / 84$ & 13 & $398-485$ & 0 & 0 & 0 & 0 & EST \\
\hline $9 / 10 / 84$ & 14 & $393-470$ & 1 & 7.1 & 0 & 0 & EST \\
\hline Dec. 84 & 56 & $362-492$ & 39 & 69.6 & 28 & 50.0 & OUI \\
\hline $14 / 03 / 85$ & 27 & $388-501$ & 6 & 22.2 & 0 & 0 & OUI \\
\hline $2 / 04 / 85$ & 26 & $372-520$ & 3 & 11.5 & 0 & 0 & OUI \\
\hline $31 / 05 / 85$ & 29 & $380-510$ & 0 & 0 & 0 & 0 & OUI \\
\hline $18 / 06 / 85$ & 24 & $400-501$ & 0 & 0 & 0 & 0 & OUI \\
\hline $23 / 07 / 85$ & 19 & $393-530$ & 6 & 31.6 & 0 & 0 & OUI \\
\hline
\end{tabular}

Tableau III : Analyses des tractus digestifs des corégones.

La gamme de tailles des poissons analysés est indiquée ( $\mathrm{mm}$ LT).

EST : des comptages n'ont pu être effectués que pour une partie des contenus stomacaux observés. Les valeurs pour l'ensemble du bol alimentaire ont èté estimées à partir de ces données.

Table III : Gut analysis of whitefish

EST : preys counted only for a part of the ingested food.

\begin{tabular}{|c|c|c|c|c|c|c|c|}
\hline DATE & $\mathbf{N}$ & $\begin{array}{l}\text { GAMME } \\
\text { DE } \\
\text { TAILLES } \\
\text { Min-Max }\end{array}$ & $\begin{array}{l}\text { T } \\
\text { ANT } \\
\text { TD } \\
\text { N }\end{array}$ & $\begin{array}{l}\text { IERS } \\
\text { SRIEURS } \\
\text { VIDES } \\
\searrow\end{array}$ & $\begin{array}{l}\mathrm{D} \\
\mathbf{N}\end{array}$ & $\begin{array}{l}\text { BES } \\
\text { STIFS } \\
\text { DES } \\
x\end{array}$ & $\begin{array}{l}\text { COMPTAGE } \\
\text { DES PROIES } \\
\text { INGEREES } \\
\text { POSSIBLE }\end{array}$ \\
\hline $\begin{array}{r}15 / 06 / 84 \\
31 / 07 / 84 \\
29 / 11 / 84 \\
20 / 12 / 84 \\
5 / 02 / 85 \\
13 / 03 / 85 \\
10 / 04 / 85 \\
25 / 04 / 85 \\
22 / 05 / 85 \\
17 / 06 / 85\end{array}$ & $\begin{array}{l}38 \\
20 \\
25 \\
30 \\
11 \\
31 \\
43 \\
30 \\
35 \\
16\end{array}$ & $\begin{array}{l}121-310 \\
142-393 \\
151-186 \\
142-188 \\
148-185 \\
146-178 \\
143-320 \\
149-195 \\
146-291 \\
150-172\end{array}$ & $\begin{array}{r}11 \\
6 \\
12 \\
15 \\
3 \\
18 \\
24 \\
3 \\
5 \\
0\end{array}$ & $\begin{array}{l}28.9 \\
30.0 \\
48.0 \\
50.0 \\
27.3 \\
58.1 \\
55.8 \\
10.0 \\
14.3 \\
0\end{array}$ & $\begin{array}{r}0 \\
0 \\
1 \\
11 \\
3 \\
10 \\
11 \\
0 \\
2 \\
0\end{array}$ & $\begin{array}{l}0 \\
0 \\
4.0 \\
36.7 \\
27.3 \\
32.3 \\
25.6 \\
0 \\
5.7 \\
0\end{array}$ & $\begin{array}{l}\text { OUI } \\
\text { OUI } \\
\text { EST } \\
\text { EST } \\
\text { OUI } \\
\text { OUI } \\
\text { OUI } \\
\text { OUI } \\
\text { OUI } \\
\text { OUI }\end{array}$ \\
\hline
\end{tabular}

Tableau IV : Analyse des tractus digestifs des gardons capturés au chalut en zone pélagique.

Table IV : Gut analysis of roach captured with trawls (pelagic area). 


\begin{tabular}{|c|c|c|c|c|c|c|c|}
\hline \multirow{2}{*}{ DATE } & \multirow{2}{*}{$\mathbf{N}$} & \multirow{2}{*}{$\begin{array}{l}\text { GAMME } \\
\text { DE } \\
\text { TAILLES } \\
\text { Min-Max }\end{array}$} & \multicolumn{2}{|c|}{$\begin{array}{c}\text { TIERS } \\
\text { ANTERIEURS }\end{array}$} & \multicolumn{2}{|c|}{$\begin{array}{c}\text { TUBES } \\
\text { DIGESTIFS } \\
\text { } \text { IDES }\end{array}$} & \multirow{2}{*}{$\begin{array}{l}\text { COMPTAGE } \\
\text { DES PROIES } \\
\text { INGEREES } \\
\text { POSSIBLE }\end{array}$} \\
\hline & & & N & $x$ & $\mathbf{N}$ & $x$ & \\
\hline Dec. 83 & 20 & $273-315$ & 12 & 60.0 & 7 & 35.0 & NON \\
\hline Fev. 84 & 31 & $153-310$ & 14 & 45.2 & 14 & 45.2 & $\mathrm{NON}$ \\
\hline $7 / 07 / 84$ & 13 & $262-320$ & 4 & 30.8 & 1 & 7.7 & OUI \\
\hline $3 / 08 / 84$ & 7 & $253-328$ & 2 & 28.6 & c & 0 & OUI \\
\hline $23 / 08 / 84$ & 11 & $276-325$ & 9 & 81.8 & 2 & 18.2 & NON \\
\hline $21 / 09 / 84$ & 12 & $191-302$ & 12 & 100 & 3 & 25.0 & NON \\
\hline $9 / 10 / 84$ & 41 & $158-326$ & 41 & 100 & 11 & 26.8 & NON \\
\hline Nov. 84 & 43 & $255-340$ & 14 & 32.6 & 2 & 4.7 & NON \\
\hline Dec. 84 & 34 & $160-386$ & 21 & 61.8 & 7 & 20.6 & NON \\
\hline Jan. 85 & 31 & $151-280$ & 29 & 93.5 & 17 & 54.8 & NON \\
\hline Fev. 85 & 21 & $165-332$ & 13 & 61.9 & 4 & 19.0 & NON \\
\hline $2 / 04 / 85$ & 25 & $160-186$ & 20 & 80.0 & 3 & 12.0 & NON \\
\hline $6 / 05 / 85$ & 16 & $271-294$ & 6 & 37.5 & 1 & 6.3 & NON \\
\hline $3 i / 05 / 85$ & 6 & $280-328$ & 1 & 16.7 & 0 & 0 & OUI \\
\hline $13 / 06 / 85$ & 14 & $160-288$ & 4 & 28.6 & 0 & 0 & OUI \\
\hline $9 / 07 / 85$ & 21 & $175-327$ & 1 & 4.8 & 0 & 0 & OUI \\
\hline $17 / 07 / 85$ & 35 & $157-215$ & 0 & 0 & 0 & 0 & OUI \\
\hline $23 / 07 / 85$ & 14 & $247-350$ & 3 & 21.4 & 2 & 14.3 & OUI \\
\hline
\end{tabular}

Tableau V : Analyse des tractus digestifs des gardons capturés à l'aide de filets maillants.

Table $V$ : Gut analysis of roach captured with gill nets.

\section{1. Évolution semi-quantitative du régime alimentaire}

Seuls seront étudiés ici les prélèvements pour lesquels un comptage des proies d'origine zooplanctonique s'est avéré possible. Ceux-ci correspondent à des périodes où la part de l'alimentation d'origine non zooplanctonique est négligeable (PONTON, 1986). Les spectres alimentaires des deux espèces de poissons pourront être comparés avec l'évolution saisonnière du zooplancton pélagique (Fig. 1).

\subsubsection{Corégones}

De juillet 1984 à mars 1985, puis à partir de juin 1985, Bythotrephes longimanus et Leptodora kindtii représentent plus des trois-quarts du volume du bol alimentaire zooplanctonique (Fig. 2). En avril et mai 1985, lorsque ces deux proies sont peu abondantes dans le milieu (Fig.1), elles soni remplacées par Daphnia sp.. Les Cyclopides ainsi que Bosmina sp, représentent toujours moins de $1 \%$ du volume du zooplancton ingéré.

\subsubsection{Gardons}

Les spectres alimentaires ont été déterminés séparément suivant que la longueur totale des poissons est inférieure ou supérieure à $220 \mathrm{~mm}$. Les premiers sont des individus appartenant à la cohorte 1982, les seconds sont nés entre 1975 et 1978 (PONTON et GERDEAUX, 1987).

L'importance de Daphnia sp. dans l'alimentation des gardons de la cohorte 1982 est bien mise en évidence (Fig. 3). Lorsque l'abondance de ce taxon diminue dans le milieu, il est remplacé par des Copépodes, principalement des Cyclopides, ainsi que Bythotrephes longimanus. L'alimentation des gardons des cohortes 1975 à 1978 se caractérise de même par une grande importance des Cladocères dans leur alimentation. (Fig. 4).

\subsection{Recouvrement des spectres alimentaires}

L'alimentation des gardons de plus de $220 \mathrm{~mm}$ (LT) est plus proche de celle des corégones que ne l'est celle des gardons nés en 1982 (Tableau VI). Les indices de similitude sont maximaux durant la période printemps-été lorsque les proies principalement ingérées, Daphnia sp. puis Bythotrephes longimanus et Leptodora kindtii, sont abondantes dans le milieu. 


\section{COTES}
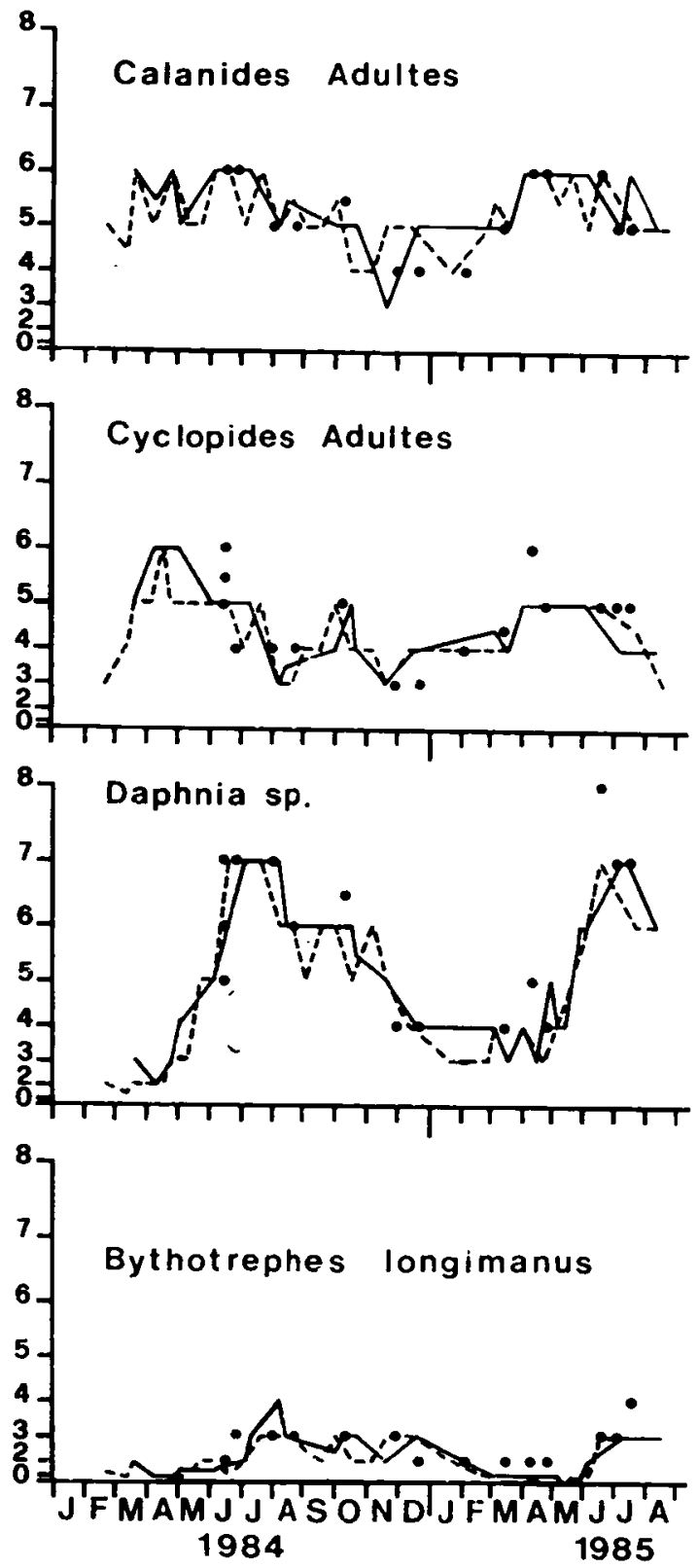

TEMPS

\section{COTES}
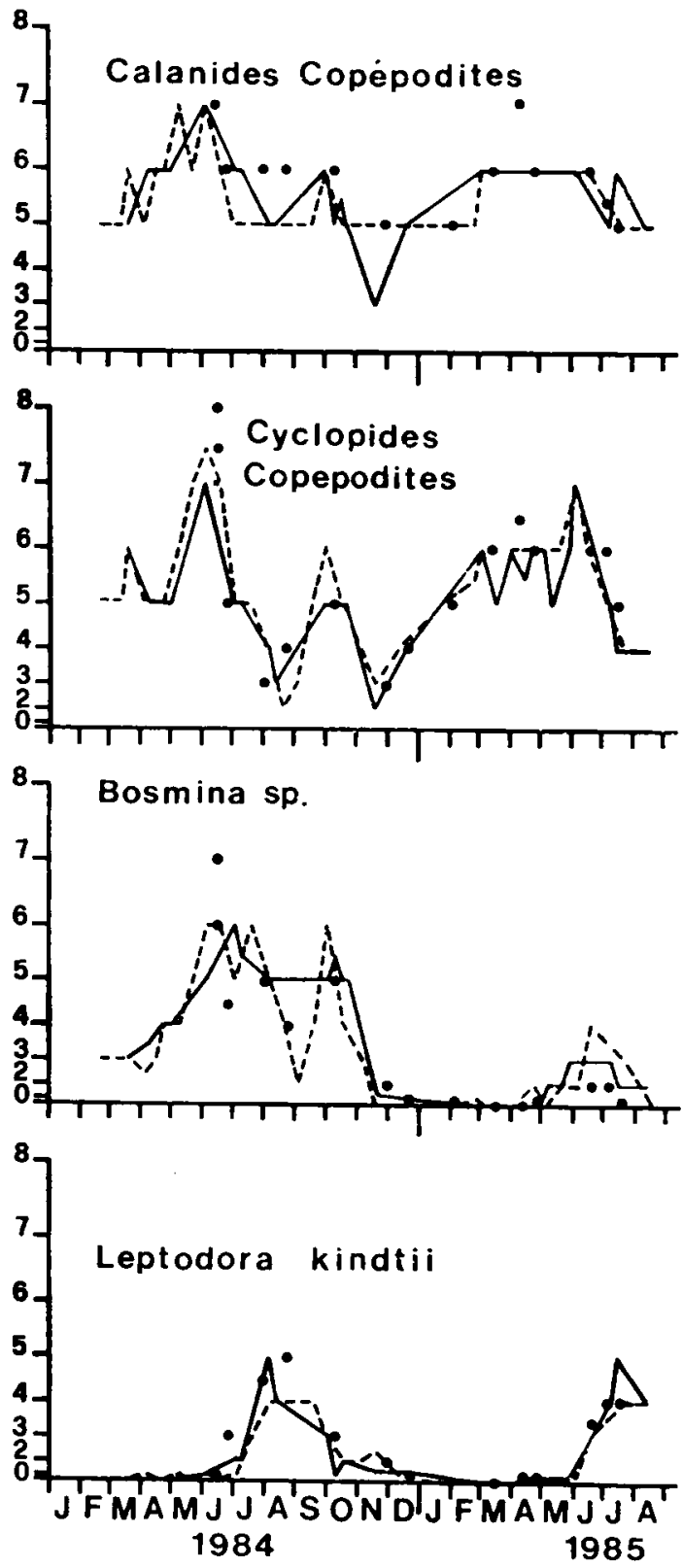

TEMPS

Figure 1: Évolution saisonnière des principaux taxons du zooplancton.

Trait plein station SHL 1 (BALVAY comm. pers.)

Trait pointille Station SHL 2 (BALVAY comm. pers.)

Points : valeurs intégrées des profils effectués a l'aide de la bouteille de 90 litres (PONTON, 1986).

Les classes d'abondance correspondant aux cotes utilisées sont celles du tableau 1. La représentation est celle préconisée par FRONTIER (1969).

Figure 1: Evolution of the principal zooplanctonic taxa.

Solid line : point SHL 1, dashed line : point SHL 2

Points : integrated values obtained with a 90 liters sampler

Definition of the indices of abundance : see table $I$. 

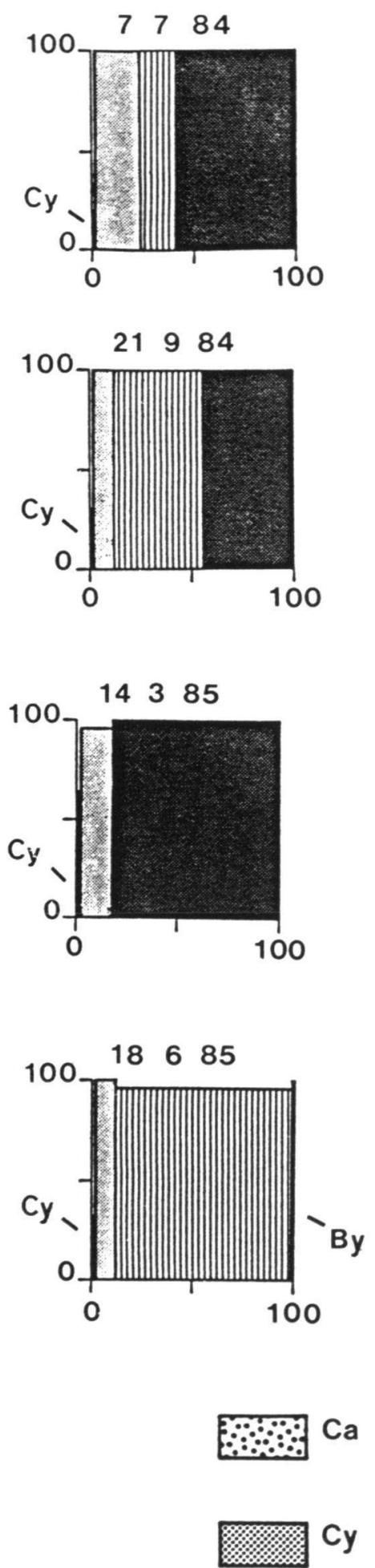
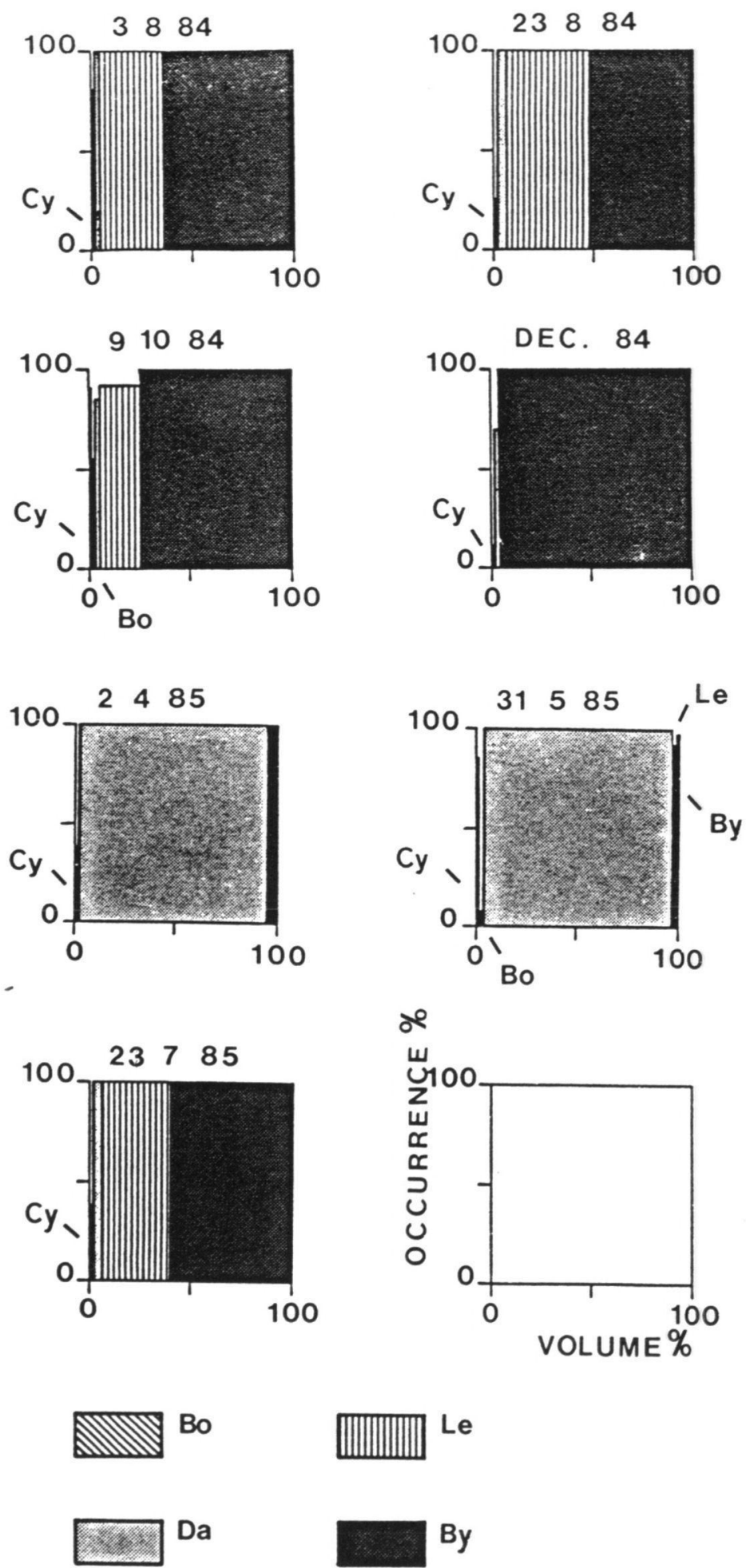

\section{y}



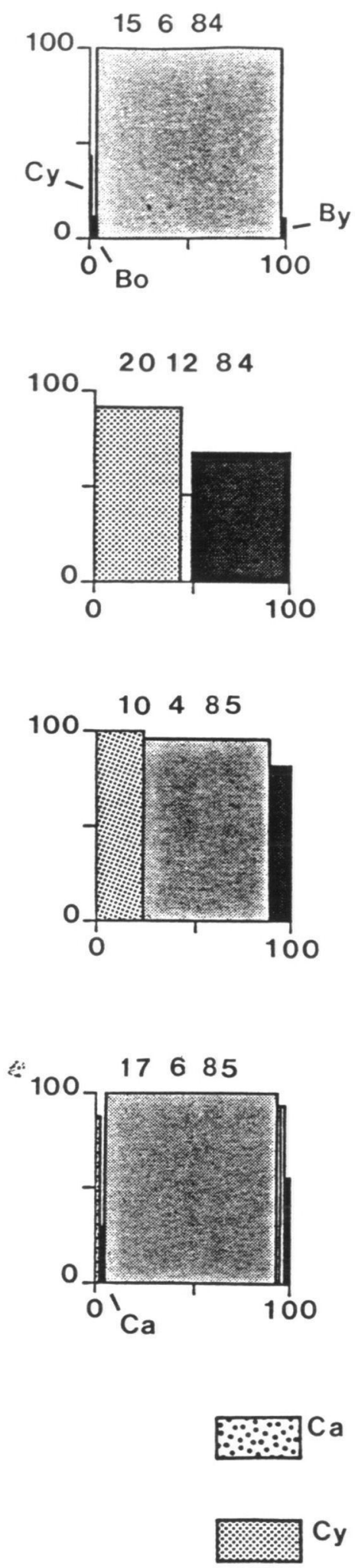

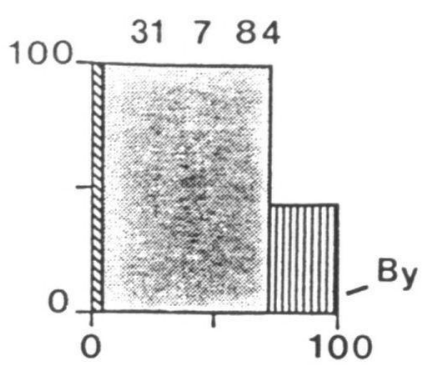

5285
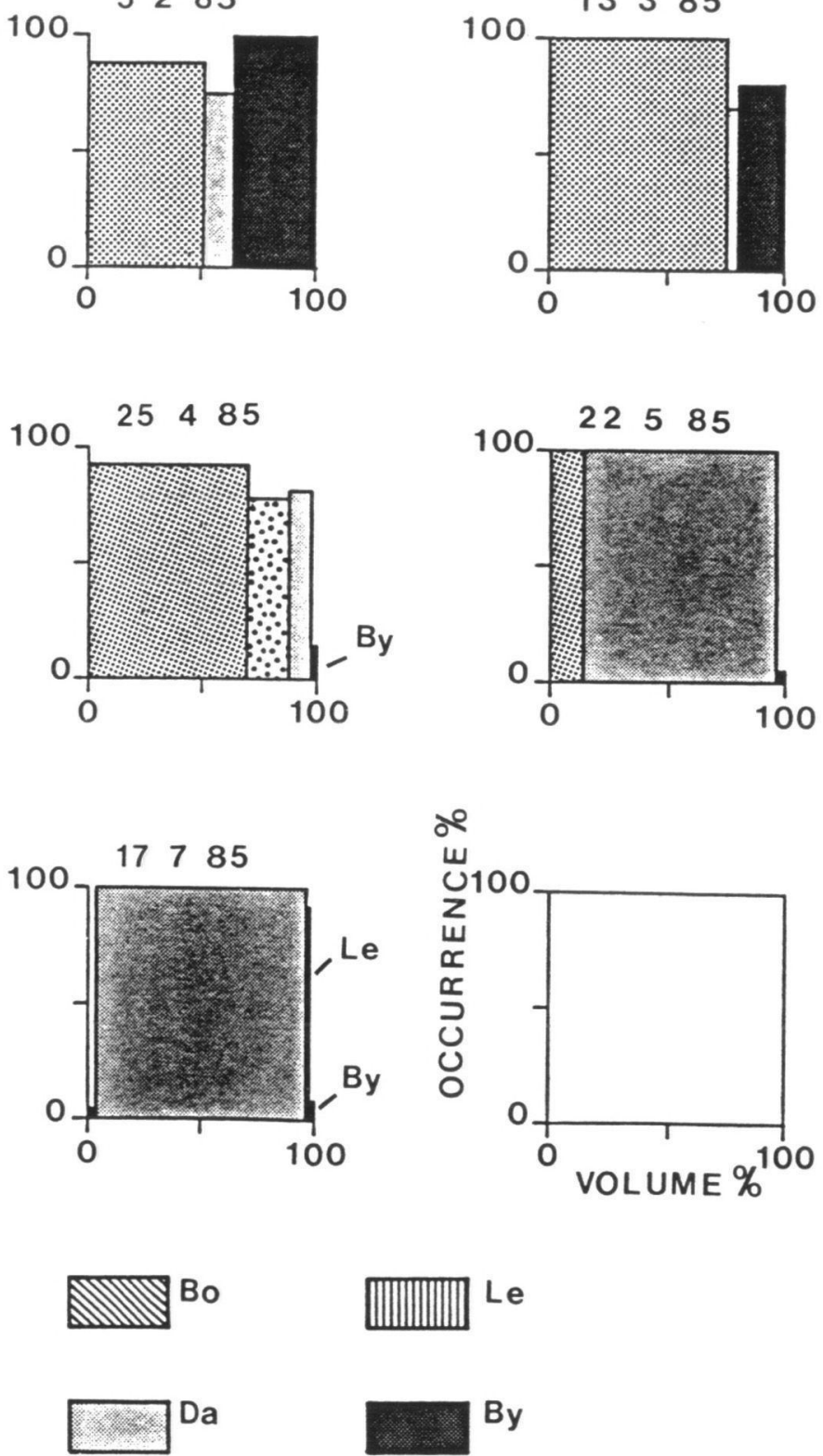

Figure 3 : Spectres alimentaires des gardons de la cohorte 1982.

Figure 3 : Zooplanctonic diet spectra of roach (year class 1982). 

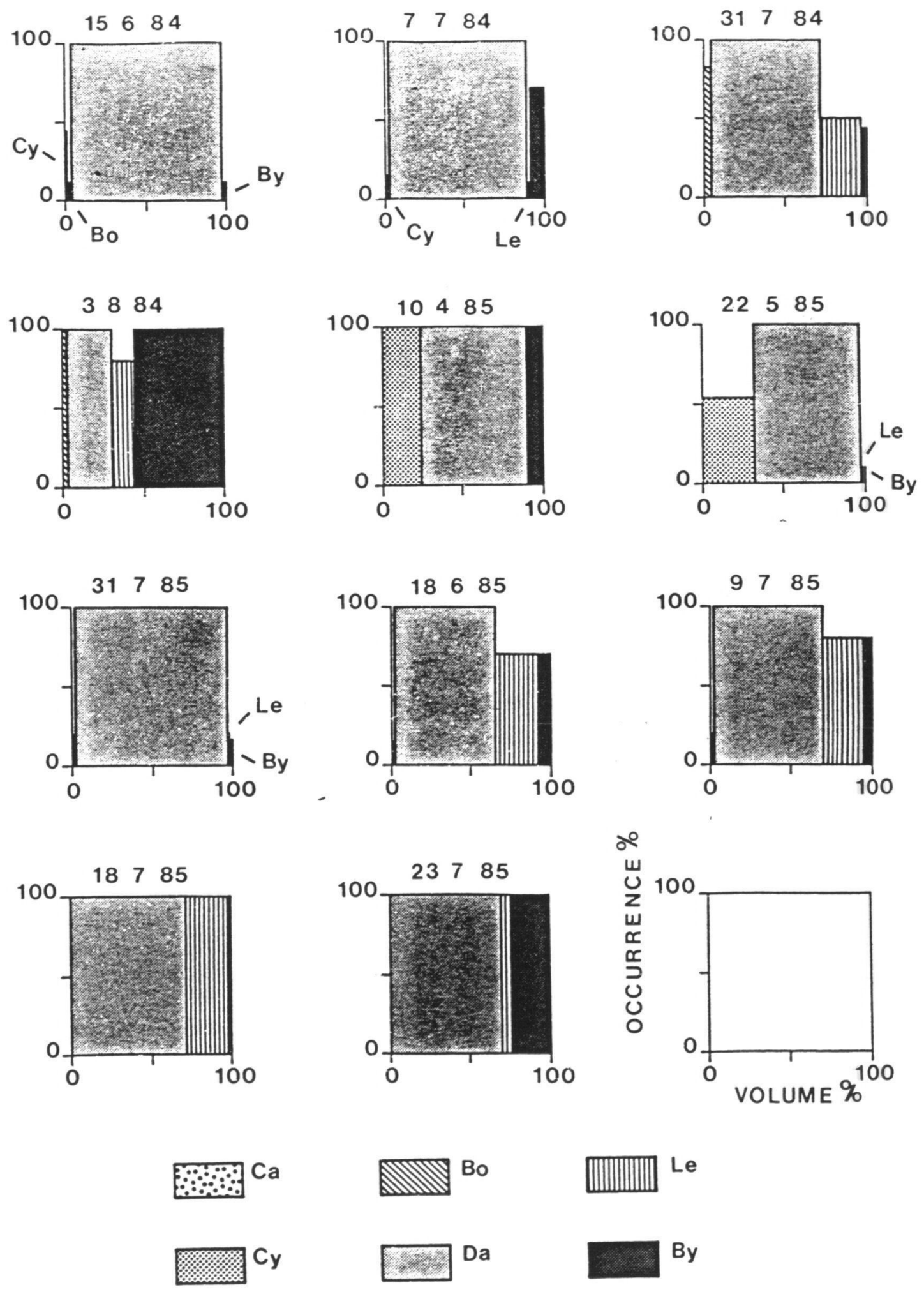

\section{By}

Figure 4: $\quad$ Spectres alimentaires des gardons des cohortes 1975 à 1978.

Figure 4 : Zooplanctonic diet spectra of roach (year classes 1975 to 1978). 
a: été 1984

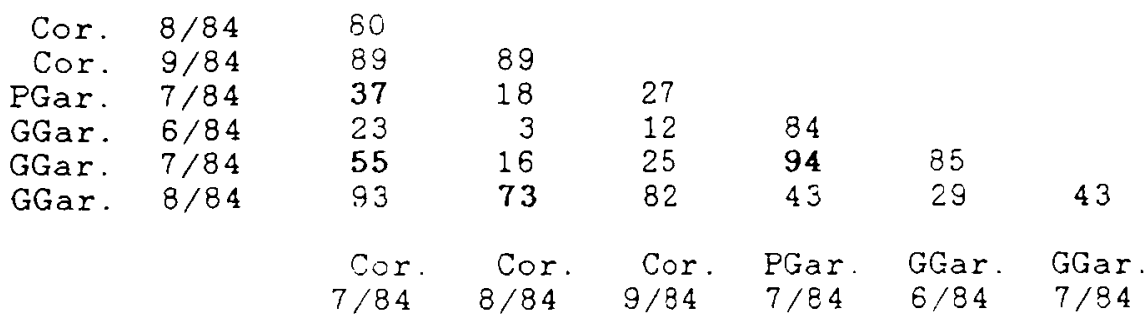

b: hiver 1984 -printemps 1985

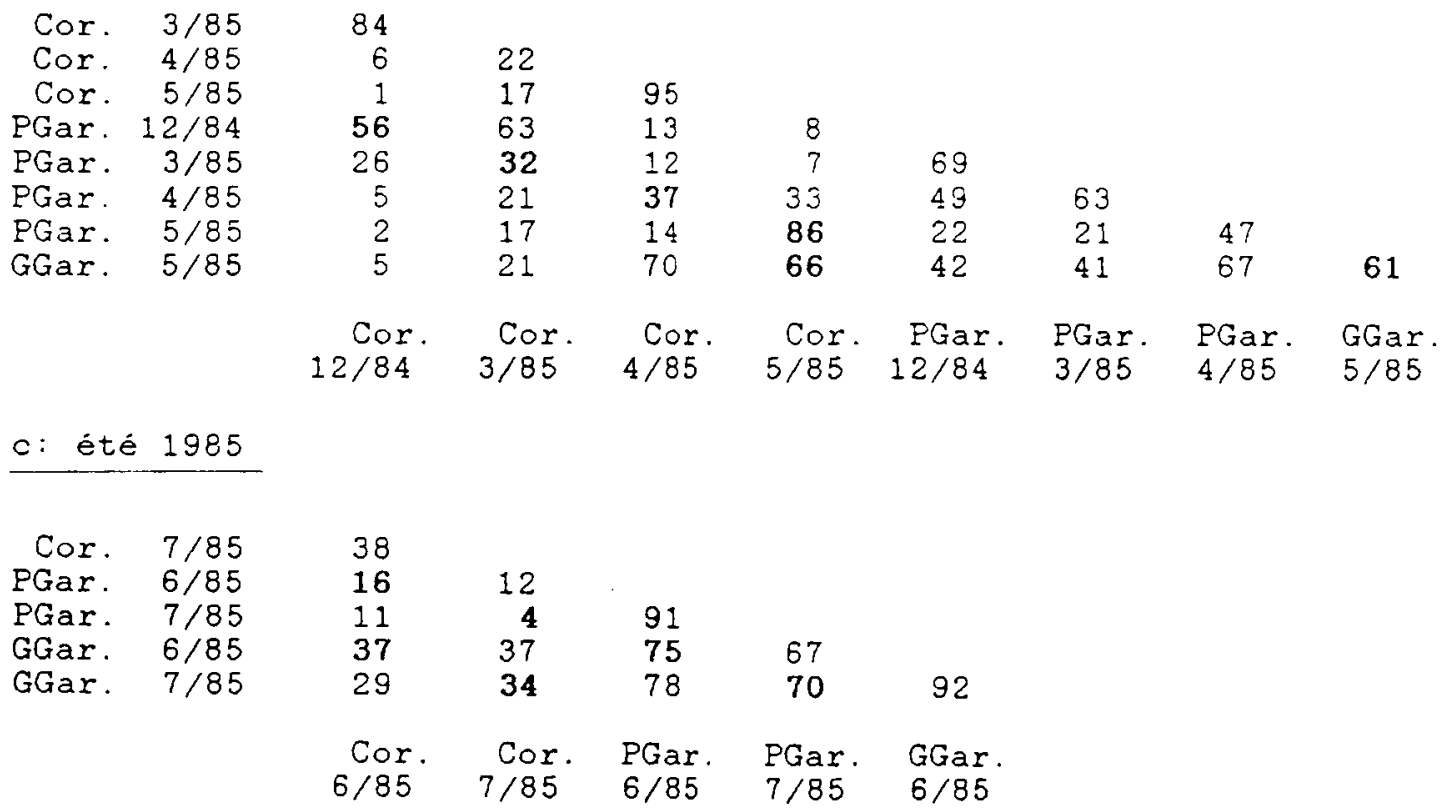

Tableau VI : Indices de similitude (en \%) des spectres alimentaires.

Cor : Corégones

PGar: Gardons de moins de $220 \mathrm{~mm}$ de longueur totale (cohorte 1982)

GGar : Gardons de plus de $220 \mathrm{~mm}$ de longueur totale (cohortes 75 a 78 )

Table VI : Similitude index $(\%)$ of diet spectra.

Cor: whitefish

PGar : roach with total length less than $220 \mathrm{~mm}$ (1982 year class)

GGar : roach with total length more than $220 \mathrm{~mm}$ (1975 to 1978 year classes).

\section{DISCUSSION}

Dans le lac Léman, les corégones de plus de $300 \mathrm{~mm}$ de longueur totale présentent, lorsqu'ils se trouvent en zone pélagique, une alimentation d'origine strictement zooplanctonique principalement dirigée vers les grandes formes du zooplancton. Ce type de spectre alimentaire se retrouve chez les corégones du lac Majeur (BERG et GRIMALDI, 1965 et 1966 ; GIUSSANI, 1974), des lacs de Thun et de Bienne (RUFLI, 1975, 1978 et 1979).

L'alimentation du gardon est bien connue dans de petits milieux et dans certaines rivières (HELLAWELL, 1972; MANN, 1973; NIEDERHOLZER et HOFER, 1980 ; PERSSON, 1983 ; GERDEAUX, 1986 ; entre autres). Les régimes alimentaires décrits dans ces milieux présentent une forte composante d'origine benthique. Les gardons du Léman sont au contraire microphages. Quel que soit leur âge, ils consomment Daphnia sp. et Bythotrephes longimanus pendant la plus grande partie de l'année. Leptodora kindtii n'est consommée qu'en été lorsque ses effectifs sont maximaux. Les Cyclopides sont plus souvent présents dans l'alimentation des jeunes individus que dans celle des plus vieux. 
Pour cette espèce, le nombre de tiers antérieurs du tube digestif vides est toujours ṫlévé chez les individus capturés à l'aide d'engins passifs et les proies sont souvent à un stade de digestion tel que tout comptage est impossible. Ce fait confirme bien l'intérêt d'engins de capture actifs pour l'étude de l'alimentation des poissons notamment pour le gardon. En effet chez cette espèce, le bol alimentaire est rapidement évacué du tiers antérieur du tube digestif (HOFER et al., 1982), partie dans laquelle le nombre de proies ingérées peut être déterminé.

On remarquera que les Calanides sont présents toute l'année en quantité importante dans le milieu. Or, peu de gardons et aucun corégone ne semblent consommer ces taxons. La faible vulnérabilité des Calanides vis-à-vis des poissons pourrait s'expliquer par une aptitude élevée de ces animaux à la fuite (DRENNER et al., 1978; DRENNER et MC COMAS, 1980).

L'étude du régime alimentaire d'un prédateur permet la détermination de sa niche alimentaire sur le plan qualitatif. Dans le but de quantifier cette niche, de nombreuses mesures de son étendue sont proposées dans la littérature scientifique. Le plus souvent, ces mesures ne tiennent pas compte de la disponibilité des ressources de l'animal étudié (HURLBERT, 1978; PETRAITIS, 1979). Elles aboutissent donc à une interprétation biologique ni appropriée, ni simple. En effet, ces ressources doivent être mesurées en biomasse en tenant compte des différences de production ou de taux de renouvellement entre les divers groupes et aussi du coût énergétique de la capture des proies ainsi que de leur valeur nutritionnelle (SCHOENER, 1974 in HURLBERT, 1978). De plus, dans l'étude comparée des niches alimentaires d'espèces sympatriques, l'unité de temps et de lieu doit être respectée et cette analyse doit être répétée à différentes saisons et pour différentes années (BARBAULT, 1981).

Lorsque tous ces impératifs sont respectés, il reste encore deux problèmes fondamentaux:

- Comment traduire la disponibilité alimentaire réellement perçue par l'animal? En d'autres termes, quelle est la représentativité d'un échantillon de zooplancton effectué avec le plus performant des appareils d'échantillonnage par rapport à la véritable disponibilité en nourriture pour le poisson zooplanctonophage?

- La perception des classes de ressources alimentaires par l'observateur est-elle enfin pertinente en comparaison de celle du prédateur étudié (PETRAITIS, 1979)?

Actuellement; face à ces problèmes, seule paraît raisonnable une étude qualitative des différences observées dans le régime alimentaire des deux poissons planctonophages. Ainsi, lorsqu'ils sont en zone pélagique, les corégones et les gardons partagent les mêmes ressources trophiques durant une période qui va de mai à octobre (proies principales ingérées: Daphnia sp., Leptodora kindtii et Bythotrephes longimanus. Les recouvrements de leur niche alimentaire durant cette période atteignent des valeurs maximales mais les proies consommées sont abondamment représentées dans le milieu. Il existe, de plus, un décalage dans le passage d'un type de proie principale à l'autre au cours du temps. Les corégones consomment par exemple Leptodora kindtii et Bythotrephes longimanus beaucoup plus tôt, beaucoup plus longtemps et en quantité plus importante que les gardons. A la vue de ces résultats, il n'est pas possible de conclure qu'il existe une compétition alimentaire entre ces deux espèces.

Le mécanisme exact du partage des ressources trophiques passe en effet par la connaissance de la biologie et de l'écologie des deux proies principales que sont Leptodora kindtii et Bythotrephes longimanus. Ainsi, les gardons et les corégones présentent dans le Léman des croissances ayant des valeurs élevées: leur nourriture doit donc se trouver en quantité très importante en période estivale. Mais, si l'on prend les chiffres obtenus à l'aide d'échantillonneurs de zooplancton intégrant toute une colonne d'eau, on arrive à des situations où le corégone a besoin de plusieurs dizaines d'heures pour remplir son estomac. C'est ce type d'observation qui a fait douter certains auteurs de la validité de leurs analyses des contenus stomacaux de corégones (HAKKARI, 1978). Or, une bouteille à prélèvements de 90 litres (AMBUHL, 1983) a permis de mettre en évidence de fortes densités ponctuelles de Leptodora kindtii et Bythotrephes longimanus dans le Léman (PONTON, 1986). A la suite des travaux de BUTORINA (1986) sur le comportement de Polyphemus pediculus, Cladocère de la même famille que Bythotrephes longimanus, on peut supposer que la répartition de ce dernier s'effectue en agrégats de faible taille comportant de nombreux individus. Après détection d'un de ces amas, les poissons trouveraient alors une nourriture abondante et facilement accessible. Ceci expliquerait aussi que malgré l'utilisation énergétique médiocre de Leptodora Kindtii et Bythotrephes longimanus par les corégones (GIUSSANI et De BERNARDI, 1977). on note une absence de modification de la composition en lipides de leurs muscles durant la période de maturation de leurs gonades (DABROWSKI et CHAMPIGNEULLE, 1986).

BERG (1970) le notait déjà, il y a plus de 15 ans, au sujet de l'étude des relations entre les populations zooplanctoniques et les poissons planctonophages : "... the problem cannot be solved if better knowledge is not gained about structure, distribution, movement of zooplancton populations on the one side, mecanisms of food assumption, rytm of food intake, vertical and horizontal movements of Coregonids on the other side". 


\section{BIBLICGRAPHIE}

AMBUHL H., 1983. Ein neuer grossvolumiger Wasserschöpfer für den Fang von Zooplankton Schweiz. Z. Hydrol., 45 (2), 498-501.

BALVAY G., DRUART J-C., PELLETIER J., PONGRATZ E., REVACLIER R., 1984. Plancton. In Le Léman. Synthèse des travaux de la CIPEL (1957-1982); CIPEL (Ed.). Lausanne, 262-313.

BARBAULT R., 1981. Ecologie des populations et des peuplements. masson, Paris, $200 \mathrm{p}$.

BERG A., 1970. A comparative study of food and growth, and competition between two species of Coregonids introduced into lake Maggiore. In: Biology of Coregonid fishes; C. C. Lindsey, C. S. Woods (Eds.). University of Manitoba Press, Winnipeg, 311-346.

BERG A., GRIMALDI E., 1965. Biologia delle due forme di coregone (Coregonus sp.) del Lago Maggiore. Mem. Ist. Ital. Idrobiol., 18, 25-196.

BERG A., GRIMALDI E., 1966. Ecological relationships between planktophagic fisk species in Lago Maggiore. Verh. int. Ver. Limmol., 16, 1065-1073.

BOHL E., 1982. Food supply and prey selection in planktivorous Cyprinidae. Oecologia, 53 (1), 134-138.

BUTORINA L. G., 1986. On the problem of aggregations of planktonic crustaceans (Polyphemus pediculus (L.). Cladocera). Arch. Hydrobiol., 105 (4), 355-386.

CHAMPIGNEULLE A., GERDEAUX D., GILLET C., 1983. Les pêches de géniteurs de corégones dans le Léman français en 1982. Bull. Fr. Piscic., 290, 149-157.

CHEVALIER Y., 1980. Le régime alimentaire du Vengeron (Rutilus rutilus (L.)) du Léman au printemps. Bull. Soc. Vaud. Sc. Nat., 357, 65-69.

DABROWSKI K. R., CHAMIGNEULLE A., 1986. Chemical composition of whitefish from Leman lake during spawning. Proc. V Congr. Europ. Ichthyol., Stockholm 1985; (sous presse).

DRENNER R. W., Mc COMAS S. R., 1980. The roles of zooplankter escape ability and size selectivity in the selective feeding and impact of planktivorous fish. In:W. C. Kerfoot (Ed.); Evolution and ecology of zooplankton communities, University Press of New England, London, 587-593.

DRENNER R. W., STRICKLER J. R., O'BRIEN W. J., 1978. Capture probability, the role of zooplankton escape in selective feeding of planktivorous Fish. Res. Bd Canada, 35, 1370-1373.

ELLIOTT J. M., 1977. Some methods for the statistical analysis of samples of benthic invertebrates Freshwater Biological Association G.B., Sci. Publ. 25, 2nd ed., 157 p.

FRONTIER S., 1969. Sur une méthode d'analyse faunistique rapide du zooplancton. J. exp. mar. Biol. Ecol., 3, 18-26.

GEIGER W., MENG H. J., MULLER R. 1985., Méthodes de recherche en biologie de pêche dans les lacs. Nouv. EAWAG, 19, 1-8.

GERDEAUX D., 1986. Écologie du gardon (Rutilus rutilus (L.)) et du sandre (Lucioperca lucioperca L.) dans le lac de Crèteil de 1977 à 1982. Étude de la ligulose du gardon. Thèse Sci. Nat., Univ. Paris VI, Paris $151 \mathrm{p}$.

GIUSSANI G., 1974. Planctofagia selettivita del coregone "Bondella" (Coregonus sp.) del lago Maggiore. Mem. Inst. Ital. Idrobiol., 31, 181-203.

GIUSSANI G., DE BERNARDI R., 1977. Food selectivity in Coregonus sp. of Lago Maggiore: an energetical approach. Mem. Ist. Ital. Idrobiol., 34, 121-130.

HAKKARI L., 1978. On the productivity and ecology of zooplankton and its role as food for fish in some lakes in central Finland. Biol. Res. Rep. Univ. Jyväskylä, 4, 3-87.

HELLAWELL J. M., 1972. The growth, reproduction and food of the roach Rutilus rutilus (L.) of the river Lugg, Herefordshire. J. Fish Biol., 4 (4), 469-486.

HOFER R., FORSTNER H., RETTENWANDER R., 1982. Duration of gut passage and its dependence on temperature and food consumption in roach Rutilus rutilus (L.): laboratory and field experiments. J. Fish Biol., 20 (3), 289-299.

HURLBERT S. H., 1978. The measurement of niche overlap and some relatives. Ecology, 59, 67-77.

JACOBSEN O. J., 1982. A review of food and feeding habits in coregonid fishes. Pol. Arch. Hydrobiol., 29 (1), 179-200).

LAUZANNE L., 1976. Régimes alimentaires et relations trophiques des poissons du lac Tchad. Cah. ORSTOM, ser. Hydrobiol., 10, 267-310.

MANN R. H. K., 1973. Observations on the age, growth, reproduction and food of the roach Rutilus rutilus (L.) in two rivers in southern England. J. Fish Biol., 5 (6), 707-736.

NIEDERHOLZER R., HOFER R., 1980. The feeding of roach (Rutilus rutilus) and rudd (Scardinius erythrophtalmus). 1 - Studies on natural populations. Ekol. Pol., 28 (1), 45-49. 
PERSSON L., 1983. Effects of intra and interspecific competition on dynamics and size structure of a Perch Perca fluviatilis and Roach Rutilus rutilus population. Oïos, 40 (1), 126-132.

PETRAITIS P. S., 1979. Likehood measures of niche breadth and overlap. Ecology, 60, 703-710.

PONTON D., 1986. Croissance et alimentation de deux poissons planctonophages du Lac Léman: le corégone (Coregonus schinzi palea Jurine) et le Gardon (Rutilus rutilus (L.)). Thèse $3^{\text {e }}$ Cycle, Univ. Lyon I, $158 \mathrm{p}$.

PONTON D. et GERDEAUX D., 1987. La population de gardons (Rutilus rutilus (L.)) du lac Léman en 1983-1985. Structure en âge, déterminisme du recrutement, analyse de la croissance. Bull. Fr. Pêche Piscic., 305, 43-53

PONTON D. et STROFFEK S., 1987. Régime alimentaire des alevins de gardons (Rutilus rutilus (L.)) dans un port du lac Leman. Comparaison avec la nourriture disponible. Schwiez. Z. Hydrol., 49 (3), (sous presse).

ROTH H., 1969. Das Weissfishproblem in der Schweiz. Veröft. Eidgen Gewässerschutz., Eidgen., Fischereiinspektion, 26, 1-69.

RUFLI H., 1975. Biologie der Coregonen in Thuner- und Bielersee. Thèse Doct. Eidgenossischen Technische Hochschule - Zuerich, $178 \mathrm{p}$.

RUFLI H., 1978. Die heutigen sympatrischen Felchenpopulationen (Coregonus spp.) des Thunerund Bielersees und ihre Morphologie. Schweiz. Z. Hydrol., 40 (1), 7-31.

RUFLI H., 1979. Ernährung und Wachstum der Felchenpopulationen (Coregonus spp.) des Thunerund Bielersees. Schweiz. Z. Hydrol., 41 (1), 64-93.

STEINBERG R., DAHM E., 1974. The use of two boat bottom midwater trawis in inland waters. Experiences in the german fishery. EIFAC/ T 23 (Suppl. 1), 23-35. 